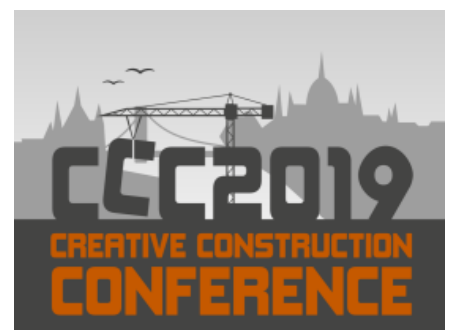

Available online at 2019.creative-construction-conference.com/proceedings/

CCC 2019

Proceedings of the Creative Construction Conference (2019) 081

Edited by: Miroslaw J. Skibniewski \& Miklos Hajdu

https://doi.org/10.3311/CCC2019-081

Creative Construction Conference 2019, CCC 2019, 29 June - 2 July 2019, Budapest, Hungary

\title{
Ontology assisted collaboration sessions on 4D BIM
}

\author{
Calin Boje $^{\mathrm{a} *}$, Veronika Bolshakova ${ }^{\mathrm{b}, \mathrm{c}}$, Annie Guerriero ${ }^{\mathrm{a}}$, Sylvain Kubicki ${ }^{\mathrm{a}}$, Gilles Halin ${ }^{\mathrm{b}, \mathrm{d}}$ \\ ${ }^{a}$ Luxembourg Institute of Science and Technology - LIST, 5 Avenue des Hauts-Forneaux, Esch-sur-Alzette L-4362, Luxemburg \\ ${ }^{b} U M R n^{\circ} 3495$ Modèles et simulations pour l'Architecture et le Patrimoine Centre de Recherche en Architecture et Ingénierie - MAP-CRAI, 1 Rue \\ Bastien Lepage, Nancy 54000, France \\ ${ }^{c}$ Centre National de la Recherche scientifique - CNRS, 3 Rue Michel-Ange, Paris 75794, France \\ ${ }^{d}$ Université de Lorraine - UL, Nancy 54000, France
}

\begin{abstract}
Stakeholders use 4D modelling during construction to coordinate project data and collaborate effectively. The collaborative sessions between project stakeholders have a complex dynamic. This research was conducted under the scope of the 4DCollab project, which aims to improve 4D BIM supported Synchronous Collaboration Sessions (SCS) by adopting a user-centric approach, whilst considering specific 4D use-case information requirements. Following several experimental SCS, an initial ontology model was developed. This model offers a holistic view of the dynamics between the following main concepts: the meeting itself (session), its participants (users), the 4D BIM model and the collaboration devices used for decision-making. Several existing schemas within the BIM domain were identified and considered for the definition of the proposed ontology, re-using several already validated concepts. The 4DCollab ontology is introduced following a rigorous design methodology. Its applications, limitations and future work are also outlined and discussed.
\end{abstract}

(C) 2019 The Authors. Published by Budapest University of Technology and Economics \& Diamond Congress Ltd.

Peer-review under responsibility of the scientific committee of the Creative Construction Conference 2019.

Keywords: 4D; BIM; Ontology; Decision-making; Collaboration; Linked Data; IfcOwl ;

\section{Introduction and research scope}

\subsection{Planning with $4 D$ BIM}

BIM processes and models are continuously present from concept design to facility management. The entire process reaches its information apex during the pre-construction and construction stages, where all stakeholder models converge in order to coordinate, collaborate and facilitate decision-making. Considering the nBIM paradigm [1], the construction stage is mainly associated with 4D BIM, where the connection between the 3D BIM and the time management information is carried out. The 4D is a digital representation of the construction site, where several usecases are applied [2] [3]. These range from construction logistics and safety management to modern site monitoring technologies which employ mobile devices, laser scanners and drones. These processes bring several heterogeneous sources of information to the decision-making, in various formats and with different levels of semantics. This research was developed under the 4DCollab project [4], which aims to deliver a novel, more holistic and collaborative 4D

*Corresponding author: Author email: calin.boje@list.lu 
Boje, Bolshakova, Guerriero, Kubicki, Halin / Proceedings of the Creative Construction Conference (2019) 081 https://doi.org/10.3311/CCC2019-081

system, while considering a user-centric approach. The scope of this article is narrowed down to the ontology representation of Synchronous Collaboration Sessions (SCS), within the context of 4D BIM and Natural User Interface (NUI) devices. The article introduces the research basis on which the 4DCollab ontology was created, a presentation of its main concepts and also its alignments with the IFC schema.

\subsection{Synchronous collaboration using natural user interfaces}

The interfaces of 4D BIM tools are traditionally aimed at trained CAD professionals. Additionally, these tools are not designed for collaborative devices. To overcome these limitations, our research explores the use of NUI to offer improved interaction between users and the 4D model during SCS. We define a SCS as the setting in which project stakeholders meet around a digital table, represented by a multi-touch collaborative device [5], with their digital documents that are brought in dynamically, according to meeting scope and needs. Experiments conducted in the past based on this setting[6], revealed several requirements in terms of: 1) user-centric interface features and 2) relevant project data. The heterogeneous project data varies from 3D geometry (IFC, laser scans, etc.), planning to site change order forms, which is used within the context of $4 \mathrm{D}$ modelling. However, these data sources are not semantically connected, hindering collaboration as a result. SCS should allow a semantically rich, knowledge-driven environment which would place model data at the fingertips of the end-users.

\subsection{Linked data paradigm and ontology representations}

The semantic web linked data paradigm allows to connect BIM with the IoT and AI agents, thus paving the way to automation, smart construction and digital twins. This study aims to benefit from linked construction data and to model the context of 4D BIM-based SCS. Although the IFC schema can be used to accurately represent 4D models, as it already includes sophisticated concepts for time and scheduling, it lacks in concepts related to inter-user collaboration and their decision-making processes. Additionally, the use of the IFC schema within the 4D domain is currently limited to research [7] with a vast majority of concepts not implemented nor used in practice.

The emergence of IfcOwl [8] has seen several implementations within a semantic web context [9]. The ontology representation of the BIM not only bypasses the interoperability problem, but also offers a more robust foundation to store and to link data on the web. Most importantly, it allows a more comprehensive conceptual and consequently digital representation of real-world 'things', from simple semantics to knowledge.

\section{4DCollab ontology design methodology}

A rigorous experiment focused methodology was employed, based on observations of conducted from seven SCS, and on the feedback of the AEC professionals involved. In order to ensure that validated concepts from adjacent knowledge domains are re-used, existing schemas and ontologies were surveyed, and their concepts considered (section 3). The ontology itself (section 4) was set to follow certain objectives in order to focus its use, as outlined in Figure 1. It is acknowledged that the process of constructing the 4DCollab ontology is iterative, and thus some concepts and their relationships are expected to change after further testing in via real use-cases.

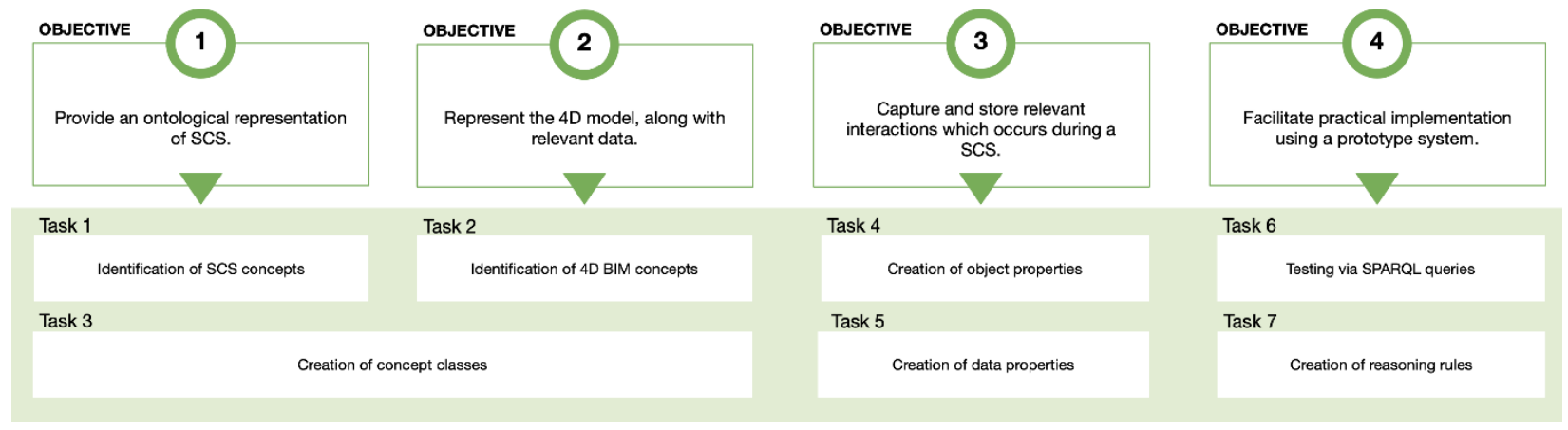

Figure 1. Methodology employed for the design and testing of 4DCollab ontology 
Boje, Bolshakova, Guerriero, Kubicki, Halin / Proceedings of the Creative Construction Conference (2019) 081 https://doi.org/10.3311/CCC2019-081

\section{Existing ontologies, schemas and workflows}

Several open schemas relevant to the BIM, collaboration and project management domains were considered throughout the 4DCollab ontology construction (Table 1). Concepts related to 3D and 4D models, Time, Collaboration, Level of Detail/Development (LOD) and Resource (document, materials, etc.) were included.

Table 1. Identified relevant ontologies classified by major concepts

\begin{tabular}{|c|c|c|c|c|c|c|c|}
\hline \multirow{2}{*}{ Ontology/Schema } & \multirow{2}{*}{ REF } & \multicolumn{5}{|c|}{ Concept fields } & \multirow[b]{2}{*}{ LOD } \\
\hline & & $3 \mathrm{D}$ & Time & $4 \mathrm{D}$ & Collaboration & Resource & \\
\hline IFC schema / IfcOwl ontology & [8] & $\checkmark$ & $\checkmark$ & $\checkmark$ & & $\checkmark$ & \\
\hline PROMONT ontology & [14] & & $\checkmark$ & & $\checkmark$ & $\checkmark$ & \\
\hline Microsoft Project schema & [11] & & $\checkmark$ & & & $\checkmark$ & \\
\hline W3C Time Ontology & [12] & & $\checkmark$ & & & & \\
\hline Collaboration Ontology $(\mathrm{CO})$ & [13] & & & & $\checkmark$ & & \\
\hline Ontology for Property Management (OPM) & [15] & & & & & & $\checkmark$ \\
\hline Building Topology Ontology (BOT) & [10] & $\checkmark$ & & & & & \\
\hline
\end{tabular}

The majority of schema models outlined in Table 1 are heavily focused on specific domains, with relatively small scopes, and are therefore relevant to only one or two field concepts. The PROMONT ontology on the other hand, represents collaboration and project management concepts in a generic field, which could be re-used to describe relationships between tasks and time management. However, its remoteness limits its usability.

The IFC schema and its ontology version are the most suitable candidates for representing the 3/4D BIM. Thus, IfcOwl can be seen as a source of model information, while ensuring interoperability with other industry tools and formats. However, the complexity and heavy structure of the IFC schema and consequently IfcOwl make it a less than optimal ontology to be used in practice, especially where reasoning is concerned. This has been a subject of research in its own right and as a response, the scientific community within the AEC/FM industry has come up with several smaller and more modular ontologies, such as BOT or OPM , most of which are aligned with IfcOwl. However, none of these newer ontology models have within scope the synchronous collaboration between users, and their interactions with various digital documents and artefacts, which were outlined as part of this research in [2].

Another important aspect that was taken into account was the collaborative meeting workflow. Existing guidance on 4D BIM modelling [16] describes the collaborative process of combining the 3D with the scheduling information, but not in a synchronous context. Additionally, various artefacts are in use during SCS, which are brought to the table in an ad-hoc manner. Therefore, one of the aims of the 4DCollab ontology was to provide the necessary relationships between these artefacts, the BIM, the actors and any other relevant ad-hoc external resources which are required in the decision-making process.

\section{4DCollab ontology}

Considering the objectives in Figure 1 from section 2, the first two refer to representing 'things' which are both virtual (the BIM) and real (the site, the actors, the devices), thus capturing an environment in which people use the 4D model to make changes and take decisions. The third is more concerned with providing an intelligent, knowledge-driven environment where the context of each session is captured for future reference and analysis, adding value to knowledge management and data analytics. The final objective is to test and validate the ontology, which would further ensure its efficacy and completeness.

The conjunction of project aims and the ontology objectives were used to design an initial 4DCollab ontology, presented in Figure 2. The main concepts are presented below, following several competency questions which were used to logically guide the scope of the ontology. The use of 'competency questions' is a formal way to assess that an ontology meets certain objectives and is capable of providing an answer to the problem it is applied to. 
Boje, Bolshakova, Guerriero, Kubicki, Halin / Proceedings of the Creative Construction Conference (2019) 081 https://doi.org/10.3311/CCC2019-081

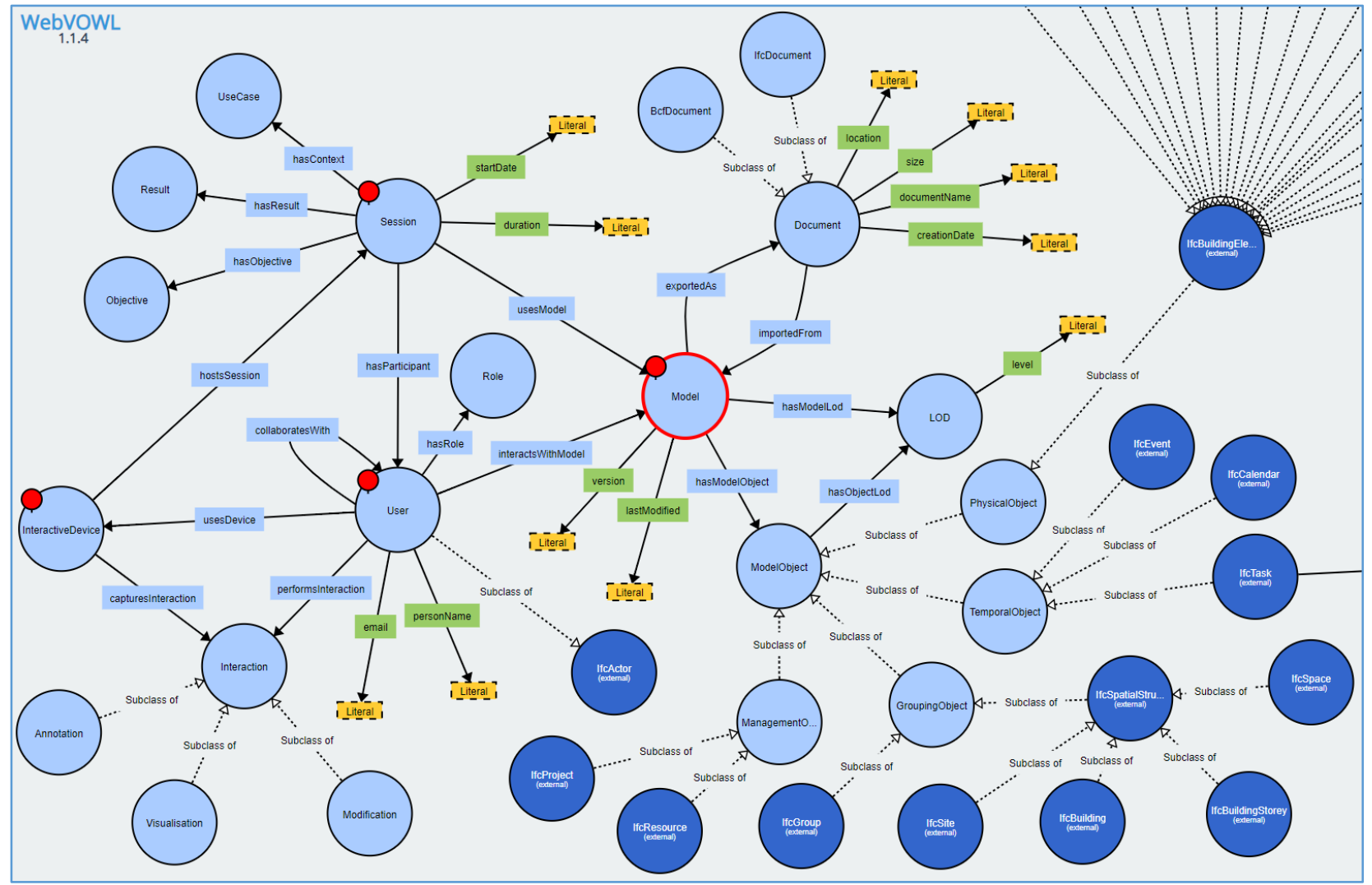

Figure 2. Overview of the 4DCollab ontology main classes with their object and data properties

\subsection{Main classes}

The core 4DCollab concepts were outlined by posing the following competency question:

"What type of 'things' does a synchronous collaborative session have?"

These concepts were broadly confined within the things around the discussion table:

- Session - the abstract concept referring to a synchronous collaborative meeting, at a certain point in time; Each session is related to a specific Model (the 4D BIM), and has specific Objectives at the beginning and Results at the end. The context of each session is dictated by the UseCase, referring to the 4D use-cases [2] [3] which filters the necessary model data for visualization and interactions.

- Model - the abstraction of the 4D model which is the subject of debate during a session, which itself comprises of 4D BIM objects, which are importedFrom documents before a session and exported at the end, as Results;

- User - representing people around the table which participate in collaborative meeting. Each User has a specific Role [2], and his Interactions with the NUI is captured by the device used to host the Session;

- InteractiveDevice - a physical collaborative device which allows users to visualize, manipulate and update the model using natural user interfaces.

\subsection{The $4 D$ Model}

A pivotal element within the meeting is the 4D model itself and its dependent digital objects and documents which are used by the users around the table. The primary competency question posed was: 
Boje, Bolshakova, Guerriero, Kubicki, Halin / Proceedings of the Creative Construction Conference (2019) 081 https://doi.org/10.3311/CCC2019-081

"What does the 4D model have to provide during a collaborative meeting?"

The Model class abstracts the interactions between 4D modelling objects and documents which are related to SCS. The difference between Model and Document is that the latter represents a static deliverable with low semantics, whereas the Model and its ModelObjects represent the fully semantic BIM objects which can be dynamically loaded and manipulated during the meeting. A good example of this is to compare a drawing document, which can only be visualized and annotated to a 3D model document file which can be interacted with at an object level.

The specific ModelObjects are further divided into several sub-classes (TemporalObject, PhysicalObject, GroupingObject and ManagementObject) and rely heavily on imported concepts from IfcOwl to correctly represent the 4D model according to common industry practice. PhysicalObject conceptualises all the elements within the BIM which have a physical representation in reality, therefore including the entire set of IfcBuildingElement class within IfcOwl. This can be summarised as the geometric model plus all the attached properties and its semantics. TemporalObject refers to concepts which are related to time and therefore used to describe the scheduling of the model, including in this case IfcOwl classes such as IfcEvent and IfcTask with all their attached concepts. GroupingObjects refer to the abstract collections and building shapes, consisting of IfcSpatialStructure and IfcGroup. They act as containers of other objects, being used to refer to specific zones, areas, spaces and levels within a building, to specific groups of scheduling tasks or a hybrid combination of objects. This ensures more dynamic grouping definitions, according to User needs.

\subsection{The data properties}

To align with the third objective, the 4DCollab ontology stores all relevant data about the model and the sessions via the data properties, as shown in green in Figure 2. These are used to create the context of the model, each session and its participants over time. At the model level, the data properties depend on the imported IFC classes, of which not all are shown Figure 2. However, the presence of Model related data will be managed using the Level of Development $(L O D)$. Tts implementation will have to rely on reasoning rules to consistently check the presence/absence of data. In return, this will allow the monitoring of the model changes in accordance to the meeting objectives and results and make a connection between LOD progression not just at a 3D level, but at a 4D BIM level. These tools enable the capability of knowledge storage, and retrieval, and a means to use AI and data analytics to further optimize and replicate a design decision when it comes to $4 \mathrm{D}$ planning.

\subsection{Example model queries}

Based on the constructed ontology model, several queries are used in Table 2 to display their application for the various objectives previously envisaged.

Table 2. Example SPARQL queries and their equivalent natural language questions

\begin{tabular}{|c|c|c|}
\hline No & Scope & Queries \\
\hline \multirow[t]{2}{*}{1} & Meeting preparation & "What are the imported documents for the current session?" \\
\hline & & $\begin{array}{l}\text { SELECT ?session ?doc WHERE }\{ \\
\text { ?model 4dOnto:importedFrom ?doc . ?session 4dOnto:usesModel ?model . ?session 4dOnto:startDate ?date } \\
\text { FILTER (?date }=\text { "29/03/2019") }\}\end{array}$ \\
\hline \multirow[t]{2}{*}{2} & Model filtering & "What are the physical model objects and their IFC IDs available within the model?" \\
\hline & & $\begin{array}{l}\text { SELECT ?object ?id WHERE \{ } \\
\text { ?object rdf:type ?class . ?object ifcowl:globalIf_IfcRoot ?guid. ?guid express:hasString ?id. } \\
\text { FILTER (?class = 4dOnto:PhysicalObject) }\}\end{array}$ \\
\hline \multirow[t]{2}{*}{3} & Interaction analysis & "Which are the annotations performed during the last session?" \\
\hline & & $\begin{array}{l}\text { SELECT ?user ?annotation ?session WHERE \{ } \\
\text { ?session 4dOnto:hasParticipant ?user . ?user 4dOnto:performsInteraction ?annotation . } \\
\text { ?session 4dOnto:startDate ?date } \\
\text { FILTER (?date }=\text { "16/06/2016") }\}\end{array}$ \\
\hline
\end{tabular}


Boje, Bolshakova, Guerriero, Kubicki, Halin / Proceedings of the Creative Construction Conference (2019) 081 https://doi.org/10.3311/CCC2019-081

Each SPARQL query during live SCS would represent the needs of the collaborative device end-users. The model data and history stored within the ontology graph database would be queried ad-hoc in order to satisfy session information requirements. Simpler questions posed are limited by the availability of information (which should be present and valid), while more complex questions would require the implementation of reasoning rules.

\section{Limitations and future work}

Due to the iterative process employed for the ontology design, its structure and classes are expected to change after practical implementation and testing. The current focus is set on re-using IfcOwl concepts, due to the scale and the interoperability the IFC schema provides. However, this can be easily extended to other relevant ontologies and domains as was shown in section 3, or any source of information on the web for enrichment of user experience.

Future work will test the ontology implementation on a new information system (under development), where it would act as the semantic information layer to connect the heterogeneous data during SCS. This would in return validate the ontology and assess its completeness.

Another perceived use to be explored is the connection of the NUI with natural language processing, whereby the interactive devices are able to understand and communicate with people around the collaborative device via vocal commands. The 4DCollab ontology would provide a semantic layer to correctly search for vocally requested data.

\section{Acknowledgements}

The authors acknowledge the financial support from Fonds National de la Recherche (FNR) Luxembourg, and Agence Nationale de la Recherche (ANR) France to the 4DCollab grant reference: 11237662 (LU) / ANR-16- CE10-000601(FR).

\section{References}

[1] L. Ding, Y. Zhou, and B. Akinci, "Building Information Modeling (BIM) application framework: The process of expanding from 3D to computable nD," Autom. Constr., vol. 46, pp. 82-93, Oct. 2014. https://doi.org/10.1016/j.autcon.2014.04.009

[2] V. Bolshakova, A. Guerriero, and G. Halin, "Identification of relevant project documents to 4D BIM uses for a synchronous collaborative decision support," in Creative Construction Conference 2018, CCC 2018, 30 June - 3 July 2018, Ljubljana, Slovenia Identification, 2018. https://doi.org/10.3311/CCC2018-134

[3] A. Guerriero, S. Kubicki, F. Berroir, and C. Lemaire, "BIM-enhanced collaborative smart technologies for LEAN construction processes," in 2017 International Conference on Engineering, Technology and Innovation (ICE/ITMC), 2017, vol. 2018-Janua, no. June, pp. 1023-1030. https://doi.org/10.1109/ICE.2017.8279994

[4] LIST, “4DCollab-project,” 2019. [Online]. Available: http://www.4dcollab-project.eu/. [Accessed: 19-Mar-2019].

[5] Shariiing, "Meetiiim collaborative working platform," 2019. [Online]. Available: https://www.shariiing.com/. [Accessed: 19-Mar-2019].

[6] V. Bolshakova, G. Halin, P. Humbert, and C. Boton, "Digital Synchronous Collaboration Workspace and 3D Interactions for an AEC Project. Decision-Making Scenario Evaluation BT - Cooperative Design, Visualization, and Engineering,” 2017, pp. 168-176. https://doi.org/10.1007/978-3-319-66805-5_21

[7] H. Hamledari, B. McCabe, S. Davari, and A. Shahi, "Automated Schedule and Progress Updating of IFC-Based 4D BIMs," J. Comput. Civ. Eng., vol. 31, no. 4, p. 04017012, Jul. 2017. https://doi.org/10.1061/(ASCE)CP.1943-5487.0000660

[8] J. Beetz, J. van Leeuwen, and B. de Vries, "IfcOWL: A case of transforming EXPRESS schemas into ontologies," Artif. Intell. Eng. Des. Anal. Manuf., vol. 23, no. 01, p. 89, Feb. 2009. https://doi.org/ 10.1017/S089006040900012

[9] P. Pauwels, S. Zhang, and Y. C. Lee, "Semantic web technologies in AEC industry: A literature overview," Autom. Constr., vol. 73, pp. 145-165, 2017. https://doi.org/10.1016/j.autcon.2016.10.003

[10] G. F. Schneider, "Towards Aligning Domain Ontologies with the Building Topology Ontology," 5th LDAC Work. 13-15 Novemb., no. April, 2017.

[11] Microsoft, "Microsoft Office Project 2007 XML Data Interchange Schema," 2019. [Online]. Available: https://docs.microsoft.com/enus/office-project/xml-data-interchange/project-xml-data-interchange-schema-reference?view=project-client-2016. [Accessed: 19-Mar2019].

[12] W3C, "Time Ontology in OWL," 2017. [Online]. Available: https://www.w3.org/TR/owl-time/. [Accessed: 19-Mar-2019].

[13] S. W. Knoll, T. Plumbaum, and J. L. Hoffmann, "Collaboration Ontology : Applying Collaboration Knowledge to a Generic Group Support System Collaboration Ontology : Applying Collaboration Knowledge to a Generic Group Support System,” no. June, 2010.

[14] S. Abels, F. Ahlemann, A. Hahn, K. Hausmann, and J. Strickmann, "PROMONT - A Project Management Ontology as a Reference for Virtual Project Organizations," vol. 2889, no. January, 2006, pp. 813-823.https://doi.org/10.1007/11915034_105

[15] M. H. Rasmussen, M. Lefrançois, M. Bonduel, C. A. Hviid, and J. Karlshø, "OPM: An ontology for describing properties that evolve over time," in CEUR Workshop Proceedings, 2018, vol. 2159, pp. 23-33.

[16] Computer Integrated Construction Research Program, "BIM Project Execution Planning Guide - Version 2.1.," May, The Pennsylvania State University, University Park, PA, USA, 2011. 